\title{
On the Rogosinski radius for holomorphic mappings and some of its applications
}

\author{
by \\ Lev Aizenberg (Ramat-Gan), Mark Elin (Karmiel) and \\ DAVID SHOIKHET (Karmiel)
}

Abstract. The well known theorem of Rogosinski asserts that if the modulus of the sum of a power series is less than 1 in the open unit disk: $\left|\sum_{n=0}^{\infty} a_{n} z^{n}\right|<1,|z|<1$, then all its partial sums are less than 1 in the disk of radius $1 / 2$ :

$$
\left|\sum_{n=0}^{k} a_{n} z^{n}\right|<1, \quad|z|<\frac{1}{2},
$$

and this radius is sharp.

We present a generalization of this theorem to holomorphic mappings of the open unit ball into an arbitrary convex domain. Other multidimensional analogs of Rogosinski's theorem as well as some applications to dynamical systems are considered.

1. Preliminaries. Let $\Omega$ be a domain in $\mathbb{C}^{n}$. By $\operatorname{Hol}(\Omega, \mathbb{C})$ we denote the space of holomorphic functions in $\Omega$ with the topology of uniform convergence on compact sets. Let $\left\{\phi_{k}(z)\right\}_{k=0}^{\infty}$ be a given basis in $\operatorname{Hol}(\Omega, \mathbb{C})$.

Definition 1.1. The Bohr radius $\mathbf{B}\left(\Omega,\left\{\phi_{k}\right\}\right)$ is the largest number $r>0$ such that if $f \in \operatorname{Hol}(\Omega)$ has the expansion

$$
\sum_{k=0}^{\infty} c_{k} \phi_{k}(z)=f(z)
$$

and if $|f(z)|<1$ in $\Omega$, then

$$
\sum_{k=0}^{\infty}\left|c_{k} \phi_{k}(z)\right|<1
$$

in $\Omega_{r}=r \Omega$, homothety of $\Omega$.

Definition 1.2. The Rogosinski radius $\mathbf{R}\left(\Omega,\left\{\phi_{k}\right\}\right)$ is the largest number $r>0$ such that, under the conditions of the previous definition, the

2000 Mathematics Subject Classification: Primary 32A05.

Key words and phrases: holomorphic mappings, power series extension, Bohr radius, Rogosinski radius, Cauchy problem, holomorphic generators. 
partial sums

$$
S_{m}(z)=\sum_{k=0}^{m} c_{k} \phi_{k}(z)
$$

satisfy the inequality $\left|S_{m}(z)\right|<1$ in $\Omega_{r}$ for all $m=0,1,2, \ldots$

Since

$$
\left|S_{m}(z)\right| \leq \sum_{k=0}^{m}\left|c_{k} \phi_{k}(z)\right| \leq \sum_{k=0}^{\infty}\left|c_{k} \phi_{k}(z)\right|,
$$

it is clear that $\mathbf{B}\left(\Omega,\left\{\phi_{k}\right\}\right) \leq \mathbf{R}\left(\Omega,\left\{\phi_{k}\right\}\right)$.

In the particular case when the domain $\Omega$ is the open unit disk $\Delta=$ $\left\{z_{1}:\left|z_{1}\right|<1\right\}$ in $\mathbb{C}^{1}$, and $\left\{\phi_{k}(z)\right\}_{k=0}^{\infty}$ is the usual power basis $\left\{z^{k}\right\}_{k=0}^{\infty}$, we denote the Bohr radius and the Rogosinski radius by $\mathbf{B}$ and $\mathbf{R}$ respectively. The following two classical results are well known.

Theorem 1.1 (Bohr, [12] see also [14]). $\mathbf{B}=1 / 3$.

Theorem 1.2 (Rogosinski [17], see also [14, 18]). $\mathbf{R}=1 / 2$.

Recently, there has been much research activity on the Bohr radius in the multidimensional case. Let us recall some of the results.

Let $U_{n}=\left\{z \in \mathbb{C}^{n}:\left|z_{j}\right|<1, j=1, \ldots, n\right\}$ be the open unit polydisk.

Theorem 1.3 (see [11]). If $\left\{z^{\alpha}\right\}$ is the power basis in $\operatorname{Hol}\left(U_{n}, \mathbb{C}\right)$, then for all $n>1$,

$$
\frac{1}{3 \sqrt{n}}<\mathbf{B}\left(U_{n},\left\{z^{\alpha}\right\}\right)<\frac{2 \sqrt{\log n}}{\sqrt{n}} .
$$

For the open unit hypercone $D_{n}^{1}=\left\{z \in \mathbb{C}^{n}:\left|z_{1}\right|+\cdots+\left|z_{n}\right|<1\right\}$ the following theorem gives sharp estimates for the Bohr radius.

Theorem 1.4 (see [2]).

$$
\frac{1}{3 \sqrt[3]{e}}<\mathbf{B}\left(D_{n}^{1},\left\{z^{\alpha}\right\}\right) \leq \frac{1}{3}
$$

Furthermore, for the domain

$$
D_{n}^{p}=\left\{z \in \mathbb{C}^{n}:\left|z_{1}\right|^{p}+\cdots+\left|z_{n}\right|^{p}<1\right\},
$$

there are generalizations of this estimate in [10] for $1 \leq p<\infty$, and in [4] for $0<p<1$.

It was shown in [6] that the Bohr radius exists if the basis $\left\{\phi_{k}(z)\right\}_{k=0}^{\infty}$ has the following two properties:

1) one of its functions is constant, for instance, $\phi_{0}(z)=1$,

2 ) there is a point $z_{0} \in \Omega$ such that $\phi_{k}\left(z_{0}\right)=0, k \geq 1$. This fact also holds for complex manifolds. 
Observe finally that for the space of solutions of second order elliptic equations with $C^{\infty}$-coefficients one can define an analogue of the Bohr radius whose existence was proved in [9]. Other results on the Bohr radius can be found in $[3,5,7,8,13]$.

There is an essential difference between the Rogosinski radius and the Bohr radius. The results mentioned above imply that the multidimensional Bohr radius (with respect to the power basis) depends on the form of the Reinhardt domain $\Omega$ considered. Boas and Khavinson's result (Theorem 1.3), for example, implies that the Bohr radius for the polydisk depends on the dimension and tends to zero as $n \rightarrow \infty$.

We will see below that in contrast to the Bohr radius, the Rogosinski radius in a sufficiently general situation does not depend on the structure of the domain nor the dimension of the space.

Therefore, it is natural to conjecture that in an infinite-dimensional Banach space there is a domain with an appropriate basis such that the Bohr radius does not exist while the Rogosinski radius does.

In this paper we present some results on a generalization of the Rogosinski radius and its multidimensional analogs.

2. A generalized theorem of Rogosinski. Denote by $\mathbb{N}_{0}$ the set of all non-negative integers: $\mathbb{N}_{0}:=\{0,1,2, \ldots\}$ and by $\mathbb{N}_{0}^{n}=\left\{\alpha=\left(\alpha_{1}, \ldots, \alpha_{n}\right)\right.$ : $\left.\alpha_{i} \in \mathbb{N}_{0}\right\}$ the set of all non-negative multi-indices.

The following theorem is a natural generalization of Theorem 1.2 to holomorphic functions on a complete Reinhardt domain in $\mathbb{C}^{n}$.

TheOREM 2.1. Let $\Omega \subset \mathbb{C}^{n}$ be a bounded complete Reinhardt domain, and let $D \subset \mathbb{C}^{1}$ be a convex domain. Suppose that $f \in \operatorname{Hol}(\Omega, D)$ is a holomorphic function on $\Omega$ with values in $D$, and

$$
f(z)=\sum_{\alpha \in \mathbb{N}_{0}^{n}} c_{\alpha} z^{\alpha}
$$

is its Taylor series. Then for all $m_{1}, \ldots, m_{n} \in \mathbb{N}_{0}^{n}$ the partial sum $S_{m_{1}, \ldots, m_{n}}$,

$$
S_{m_{1}, \ldots, m_{n}}(z):=\sum_{\alpha_{1} \leq m_{1}, \ldots, \alpha_{n} \leq m_{n}} c_{\alpha} z^{\alpha},
$$

maps $\frac{1}{2} \Omega$ into $D$.

For holomorphic mappings between two complex Banach spaces $X$ and $Y$ a generalization of the Rogosinski Theorem can be formulated. Recall that the mapping $f: \Omega \rightarrow Y, \Omega \subset X$, is called holomorphic in $\Omega$ if $f$ is Fréchet differentiable at each point $z \in \Omega$, i.e., there exists a bounded linear operator $A(=A(z))$ such that 


$$
\lim _{\|v\| \rightarrow 0} \frac{\|f(z+v)-f(z)-A v\|}{\|v\|}=0 .
$$

The set of all holomorphic mappings in $\Omega$ taking values in a domain $D \subset Y$ is denoted by $\operatorname{Hol}(\Omega, D)$. Furthermore, any mapping $f$ holomorphic in a complete circular (balanced) domain admits there a series representation

$$
f(z)=\sum_{k=0}^{\infty} P_{k}(z)
$$

where $P_{k}$ is a homogeneous polynomial of degree $k$.

TheOREM 2.2. Let $X$ and $Y$ be complex Banach spaces. Let $\Omega \subset X$ be a bounded, complete, circular domain and $D \subset Y$ be a convex domain. Suppose $f \in \operatorname{Hol}(\Omega, D)$ is a holomorphic mapping represented by a series of homogeneous polynomials (2.3). Then for each $n \in \mathbb{N}$ the partial sum $S_{n}$,

$$
S_{n}(z):=\sum_{k=0}^{n} P_{k}(z)
$$

maps $\frac{1}{2} \Omega$ into $D$.

Note that Theorem 2.2 does not cover Theorem 2.1 because the sums (2.2) are not of the form (2.4).

The main result of this paper (Theorem 2.3 below) yields both Theorem 2.1 and Theorem 2.2. To formulate it we need some additional notions and notations.

Let $X_{1}, \ldots, X_{n}$ be complex Banach spaces equipped with the norms $\|\cdot\|_{1}, \ldots,\|\cdot\|_{n}$, respectively. We say that a domain $\Omega \subset X_{1} \times \cdots \times X_{n}$ is a generalized complete Reinhardt domain if $x=\left(x_{1}, \ldots, x_{n}\right) \in \Omega$ implies that $y \in \Omega$ for each $y=\left(y_{1}, \ldots, y_{n}\right)$ with $\left\|y_{1}\right\|_{1} \leq\left\|x_{1}\right\|_{1}, \ldots,\left\|y_{n}\right\|_{n} \leq\left\|x_{n}\right\|_{n}$. It is easy to see that any mapping $f \in \operatorname{Hol}(\Omega, Y)$ holomorphic in $\Omega$ and taking values in another Banach space $Y$ can be represented by the series

$$
f(z)=\sum_{\alpha \in \mathbb{N}_{0}^{n}} P_{\alpha}(z)=\sum_{\alpha \in \mathbb{N}_{0}^{n}} P_{\alpha_{1}, \ldots, \alpha_{n}}\left(z_{1}, \ldots, z_{n}\right),
$$

where $P_{\alpha_{1}, \ldots, \alpha_{n}}\left(z_{1}, \ldots, z_{n}\right)$ is a $Y$-valued polynomial $\alpha_{i}$-homogeneous with respect to $z_{i} \in X_{i}$.

Let $\mathcal{A}$ be a (finite or infinite) lattice in $\mathbb{N}_{0}^{n}$. We say that $\mathcal{A}$ is admissible if it can be represented as follows:

$$
\mathcal{A}=\left\{\alpha \in \mathbb{N}_{0}^{n}: m_{1 j} \alpha_{1}+m_{2 j} \alpha_{2}+\cdots+m_{n j} \alpha_{n} \leq m_{j}, j=1, \ldots, l\right\},
$$

where for each $j$ all numbers $m_{1 j}, m_{2 j}, \ldots, m_{n j}, m_{j}$ belong to $\mathbb{N}_{0}$ and have no common divisor. 
Theorem 2.3. Let $X_{1}, \ldots, X_{n}$ and $Y$ be complex Banach spaces, let $\Omega \subset X_{1} \times \cdots \times X_{n}$ be a generalized complete Reinhardt domain, and let $D \subset Y$ be a convex domain. Suppose that $f \in \operatorname{Hol}(\Omega, D)$ is represented by the series

$$
f(z)=\sum_{\alpha \in \mathbb{N}_{0}^{n}} P_{\alpha}(z)
$$

Let $\mathcal{A}$ be an admissible lattice in $\mathbb{N}_{0}^{n} \subset \mathbb{Z}^{n}$ which is defined by (2.6). Then the value of the polynomial

$$
P_{\mathcal{A}}(w):=\sum_{\alpha \in \mathcal{A}} P_{\alpha}(w)
$$

is in $D$ whenever $w$ belongs to the domain $\Omega_{\mathcal{A}}$ defined as follows:

$$
\left\{w \in X_{1} \times \cdots \times X_{n}:\left(\frac{w_{1}}{\prod_{j=1}^{l}\left(r_{m_{j}}\right)^{m_{1 j}}}, \ldots, \frac{w_{n}}{\prod_{j=1}^{l}\left(r_{m_{j}}\right)^{m_{n j}}}\right) \in \Omega\right\},
$$

where $r_{1}=1 / 2, r_{2}=\sqrt{3 / 8}$ and for $m \geq 3$ the number $r_{m}$ is the unique positive solution of the equation

$$
1-r-2 r^{m+1}=0
$$

Moreover,

$$
1-\frac{\ln m}{m}<r_{m}<1-\frac{1}{m}, \quad m \geq 3 .
$$

Thus $r_{m} \rightarrow 1$ as $m$ tends to infinity.

Proof. We prove the theorem step by step. First, we prove

SteP 1. For any $m \in \mathbb{N}, m \geq 3$, the equation (2.9) has a unique positive solution $r_{m}$ which satisfies the inequality (2.10).

Since the function $y_{1}(r)=1-r$ is decreasing on $\mathbb{R}^{+}:=(0, \infty)$ and the function $y_{2}(r)=2 r^{m+1}$ is increasing, the equation (2.9) has a unique solution $r_{m} \in(0,1)$. The right-hand inequality of (2.10) follows because $y_{2}(1-1 / m) \geq y_{1}(1-1 / m)$. To show that the left inequality of $(2.10)$ holds, one verifies that

$$
y_{1}\left(1-\frac{\ln m}{m}\right) \geq y_{2}\left(1-\frac{\ln m}{m}\right)
$$

or, equivalently,

$$
\frac{1}{2} \geq \frac{m}{\ln m}\left(1-\frac{\ln m}{m}\right)^{m+1} .
$$

Substituting for $m$ a continuous variable $t$ and setting $x=t / \ln t$, we consider the function $y(x)=x(1-1 / x)^{t(x)+1}$. Direct calculation yields for $t=3$ the 
value $x_{0}=3 / \ln 3$. Hence $y\left(x_{0}\right) \approx 0.4406<1 / 2$. Now we show that $y(x)$ decreases when $t \geq 3$. Indeed,

$$
\begin{aligned}
\frac{y^{\prime}(x)}{y(x)} & =\frac{1}{x}+t_{x}^{\prime} \ln \left(1-\frac{1}{x}\right)+\frac{t+1}{x^{2}-x} \\
& \leq \frac{1}{x}-t_{x}^{\prime}\left(\frac{1}{x}+\frac{1}{2 x^{2}}\right)+\frac{t+1}{x^{2}-x}=\frac{2 x(x+t)-t_{x}^{\prime}(2 x+1)(x-1)}{2\left(x^{3}-x^{2}\right)} \\
& =\frac{\frac{2 t^{2}}{\ln ^{2} t}+\frac{2 t^{2}}{\ln t}-\frac{\ln ^{2} t}{\ln t-1}\left(\frac{2 t}{\ln t}+1\right)\left(\frac{t}{\ln t}-1\right)}{2\left(x^{3}-x^{2}\right)} \\
& =\frac{t \ln ^{3} t+\ln ^{4} t-2 t^{2}}{2 \ln ^{2} t(\ln t-1)\left(x^{3}-x^{2}\right)} \\
& =\frac{\left(\operatorname{tn}^{3} t-\left(2-\frac{16}{e^{4}}\right) t^{2}\right)+\left(\ln ^{4} t-\frac{16}{e^{4}} t^{2}\right)}{2 \ln ^{2} t(\ln t-1)\left(x^{3}-x^{2}\right)}<0,
\end{aligned}
$$

because each summand in brackets is less than zero. Thus $y$ is a decreasing function, and this completes the proof of the first step.

STEP 2. Let $g_{m, r}(z)=\sum_{k=0}^{m} r^{k} z^{k}, z \in \Delta, r \in \mathbb{R}$. Then

$$
\operatorname{Re} g_{m, r}(z) \geq \frac{1}{2}
$$

for all $z \in \Delta$ and $r \in\left(0, r_{m}\right)$, where $r_{1}=1 / 2, r_{2}=\sqrt{3 / 8}$, and $r_{m}$ for $m \geq 3$ is the unique positive solution of the equation (2.9) on the interval $(0,1)$.

Indeed, by the maximum principle we have to show that

$$
\operatorname{Re} 2 g_{m, r}(z)-1>0 \quad \text { for any } z \in \partial \Delta \text {. }
$$

Since $|z|=1$ one can write, for $r>0$,

$$
\begin{aligned}
\operatorname{Re} 2 g_{m, r}(z)-1 & =\operatorname{Re} \frac{1-2 r^{m+1} z^{m+1}+r z}{1-r z} \\
& =\frac{1}{|1-r z|^{2}} \operatorname{Re}\left\{\left(1-2 r^{m+1} z^{m+1}+r z\right)(1-r \bar{z})\right\} \\
& =\frac{1}{|1-r z|^{2}} \operatorname{Re}\left\{1-2 r^{m+1} z^{m+1}+2 r^{m+2} z^{m}-r^{2}\right\} .
\end{aligned}
$$

In particular, for $m=1$ and $m=2$ using standard calculus one can find the minimal values of the function $1-2 r^{m+1} z^{m+1}+2 r^{m+2} z^{m}-r^{2}$ on the unit circle $|z|=1$, and then we find that $\operatorname{Re} 2 g_{1, r}(z)-1>0$ whenever $r<1 / 2$, as well as $\operatorname{Re} 2 g_{2, r}(z)-1>0$ whenever $r<\sqrt{3 / 8}$. 
For $m \geq 3$ we have

$$
\begin{aligned}
\operatorname{Re} 2 g_{m, r}(z)-1 & \geq \frac{1}{|1-r z|^{2}}\left\{1-2 r^{m+1}-2 r^{m+2}-r^{2}\right\} \\
& =\frac{1+r}{|1-r z|^{2}}\left(1-r-2 r^{m+1}\right) .
\end{aligned}
$$

Therefore, our claim follows by the first step.

SteP 3. Let $X_{1}, \ldots, X_{n}$ and $Y$ be complex Banach spaces, $\Omega \subset X_{1} \times$ $\cdots \times X_{n}$ be a generalized complete Reinhardt domain, and $D \subset Y$ be a convex domain. Then for any mapping $f \in \operatorname{Hol}(\Omega, D)$ represented by the series

$$
f(z)=\sum_{\alpha \in \mathbb{N}_{0}^{n}} P_{\alpha}(z),
$$

for each $z \in \Omega$ and for all numbers $m_{1}, m_{2}, \ldots, m_{n}, m \in \mathbb{N}_{0}$,

$$
\begin{aligned}
\oint_{|\zeta|=1} f\left(\zeta^{m_{1}} z_{1}, \ldots, \zeta^{m_{n}} z_{n}\right)\left(1+2 \operatorname{Re} \sum_{k=1}^{m} r^{k} \zeta^{k}\right) \frac{d \zeta}{2 \pi i \zeta} \\
=\sum_{\alpha: m_{1} \alpha_{1}+\cdots+m_{n} \alpha_{n} \leq m} P_{\alpha_{1}, \ldots, \alpha_{n}}\left(r^{m_{1}} z_{1}, \ldots, r^{m_{n}} z_{n}\right) .
\end{aligned}
$$

To prove this we just calculate:

$$
\begin{aligned}
\oint_{|\zeta|=1} f\left(\zeta^{m_{1}} z_{1}, \ldots, \zeta^{m_{n}} z_{n}\right)\left(1+2 \operatorname{Re} \sum_{k=1}^{m} r^{k} \zeta^{k}\right) \frac{d \zeta}{2 \pi i \zeta} \\
\quad=\oint_{|\zeta|=1}\left(\sum_{\alpha \in \mathbb{N}_{0}^{n}} \zeta^{m_{1} \alpha_{1}+\cdots+m_{n} \alpha_{n}} P_{\alpha}(z)\right)\left(1+\sum_{k=1}^{m} r^{k} \zeta^{k}+\sum_{k=1}^{m} r^{k} \bar{\zeta}^{k}\right) \frac{d \zeta}{2 \pi i \zeta} \\
=\sum_{\alpha \in \mathbb{N}_{0}^{n}} P_{\alpha}(z) \oint_{|\zeta|=1} \zeta^{m_{1} \alpha_{1}+\cdots+m_{n} \alpha_{n}} \cdot \sum_{k=0}^{m} r^{k} \bar{\zeta}^{k} \frac{d \zeta}{2 \pi i \zeta} \\
=\sum_{k=0}^{m} r^{k} \sum_{\alpha: m_{1} \alpha_{1}+\cdots+m_{n} \alpha_{n}=k} P_{\alpha}(z) \\
=\sum_{\alpha: m_{1} \alpha_{1}+\cdots+m_{n} \alpha_{n} \leq m} P_{\alpha}\left(r^{m_{1}} z_{1}, \ldots, r^{m_{n}} z_{n}\right) .
\end{aligned}
$$

STEP 4. By Step 2,

$$
1+2 \operatorname{Re} \sum_{k=1}^{m} r^{k} \zeta^{k} \geq 0
$$


for all $\zeta \in \partial \Delta$ when $r \leq r_{m}$. So, for each natural number $m$,

$$
\left(1+2 \operatorname{Re} \sum_{k=1}^{m}\left(r_{m}\right)^{k} \zeta^{k}\right) \frac{d \zeta}{2 \pi i \zeta}
$$

is a probability measure on the unit circle $\partial \Delta$. Therefore for all $z \in \Omega$ and for each $j=1, \ldots, l$ the integral

$$
\oint_{|\zeta|=1} f\left(\zeta^{m_{1 j}} z_{1}, \ldots, \zeta^{m_{n j}} z_{n}\right)\left(1+2 \operatorname{Re} \sum_{k=1}^{m_{j}}\left(r_{m_{j}}\right)^{k} \zeta^{k}\right) \frac{d \zeta}{2 \pi i \zeta}
$$

takes values in $D$. By Step 3 we have

$$
\begin{aligned}
\oint_{|\zeta|=1} f & \left(\zeta^{m_{11}} z_{1}, \ldots, \zeta^{m_{n 1}} z_{n}\right)\left(1+2 \operatorname{Re} \sum_{k=1}^{m_{1}}\left(r_{m_{1}}\right)^{k} \zeta^{k}\right) \frac{d \zeta}{2 \pi i \zeta} \\
= & \sum_{\alpha: m_{11} \alpha_{1}+\cdots+m_{n 1} \alpha_{n} \leq m_{1}} P_{\alpha_{1}, \ldots, \alpha_{n}}\left(\left(r_{m_{1}}\right)^{m_{11}} z_{1}, \ldots,\left(r_{m_{1}}\right)^{m_{n 1}} z_{n}\right) \\
= & \sum_{\alpha: m_{11} \alpha_{1}+\cdots+m_{n 1} \alpha_{n} \leq m_{1}} P_{\alpha}\left(w_{1}^{[1]}, \ldots, w_{n}^{[1]}\right),
\end{aligned}
$$

where $w^{[1]}=\left(w_{1}^{[1]}, \ldots, w_{n}^{[1]}\right)$ with $w_{i}^{[1]}=\left(r_{m_{1}}\right)^{m_{i 1}} z_{i}$. In other words,

$$
z=\left(\frac{w_{1}^{[1]}}{\left(r_{m_{1}}\right)^{m_{11}}}, \ldots, \frac{w_{n}^{[1]}}{\left(r_{m_{1}}\right)^{m_{n 1}}}\right) \in \Omega .
$$

The second integration gives

$$
\begin{gathered}
\oint_{|\zeta|=1}\left(\sum_{\alpha: m_{11} \alpha_{1}+\cdots+m_{n 1} \alpha_{n} \leq m_{1}} P_{\alpha}\left(\zeta^{m_{12} 2} w_{1}^{[1]}, \ldots, \zeta^{m_{n 2}} w_{n}^{[1]}\right)\right) \\
\times\left(1+2 \operatorname{Re} \sum_{k=1}^{m_{2}}\left(r_{m_{2}}\right)^{k} \zeta^{k}\right) \frac{d \zeta}{2 \pi i \zeta} \\
=\sum_{\alpha:\left\{\begin{array}{l}
m_{11} \alpha_{1}+\cdots+m_{n 1} \alpha_{n} \leq m_{1} \\
m_{12} \alpha_{1}+\cdots+m_{n 2} \alpha_{n} \leq m_{2}
\end{array}\right.} P_{\alpha}\left(\left(r_{m_{2}}\right)^{m_{12}} w_{1}^{[1]}, \ldots,\left(r_{m_{2}}\right)^{m_{n 2}} w_{n}^{[1]}\right) \\
=\sum_{\alpha:\left\{\begin{array}{l}
m_{11} \alpha_{1}+\cdots+m_{n 1} \alpha_{n} \leq m_{1} \\
m_{12} \alpha_{1}+\cdots+m_{n 2} \alpha_{n} \leq m_{2}
\end{array}\right.} P_{\alpha}\left(w_{1}^{[2]}, \ldots, w_{n}^{[2]}\right),
\end{gathered}
$$

where $w^{[2]}=\left(w_{1}^{[2]}, \ldots, w_{n}^{[2]}\right)$ with $w_{i}^{[2]}=\left(r_{m_{2}}\right)^{m_{i 2}} w_{i}^{[1]}$. In other words,

$$
z=\left(\frac{w_{1}^{[2]}}{\left(r_{m_{1}}\right)^{m_{11}}\left(r_{m_{2}}\right)^{m_{12}}}, \ldots, \frac{w_{n}^{[2]}}{\left(r_{m_{1}}\right)^{m_{n 1}}\left(r_{m_{2}}\right)^{m_{n 2}}}\right) \in \Omega .
$$

Integrating $l$ times we achieve the polynomial $P_{\mathcal{A}}$ and the domain $\Omega_{\mathcal{A}}$. The proof of the theorem is complete. 
In the following corollaries we consider some of the most important situations. In the first one we consider lattices of the form

$$
\mathcal{A}=\left\{\alpha \in \mathbb{N}_{0}^{n}: \alpha_{j} \leq m_{j}, j=1, \ldots, n\right\} .
$$

Corollary 2.1. Let $X_{1}, \ldots, X_{n}$ and $Y$ be complex Banach spaces, let $\Omega \subset X_{1} \times \cdots \times X_{n}$ be a generalized complete Reinhardt domain, and let $D \subset Y$ be a convex domain. Suppose that $f \in \operatorname{Hol}(\Omega, D)$ is represented by the series

$$
f(z)=\sum_{\alpha \in \mathbb{N}_{0}^{n}} P_{\alpha}(z)
$$

Then

$$
P_{m_{1}, \ldots, m_{n}}(w):=\sum_{\alpha_{j} \leq m_{j}} P_{\alpha}(w)
$$

is in $D$ whenever $w$ belongs to the domain

$$
\Omega_{m_{1}, \ldots, m_{n}}=\left\{w \in X_{1} \times \cdots \times X_{n}:\left(\frac{w_{1}}{r_{m_{1}}}, \ldots, \frac{w_{n}}{r_{m_{n}}}\right) \in \Omega\right\},
$$

where $r_{1}=1 / 2, r_{2}=\sqrt{3 / 8}$ and for $m \geq 3$ the number $r_{m}$ is the unique positive solution of (2.9).

REMARK. The domain $\Omega_{m_{1}, \ldots, m_{n}}$ defined by (2.12) may be considered a "norm ellipsoid". For example, if $\Omega \subset \mathbb{C}^{n}$ is the unit ball, $\Omega=\left\{z \in \mathbb{C}^{n}\right.$ : $\left.\sum_{j=1}^{n}\left|z_{j}\right|^{2}<1\right\}$, then (2.12) is the Euclidean ellipsoid

$$
\Omega_{m_{1}, \ldots, m_{n}}=\left\{z \in \mathbb{C}^{n}: \sum_{j=1}^{n}\left|z_{j}\right|^{2} /\left(r_{m_{j}}\right)^{2}<1\right\} .
$$

Furthermore, if $\Omega \subset \mathbb{C}^{n}$ is the unit polydisk, $\Omega=\left\{z \in \mathbb{C}^{n}:\left|z_{j}\right|<1\right\}$, then the domain (2.12) is also a polydisk: $\Omega_{m_{1}, \ldots, m_{n}}=\left\{z \in \mathbb{C}^{n}:\left|z_{j}\right|<r_{j}\right\}$.

In the following assertion we set $n=1$. Then any admissible lattice has the form

$$
\mathcal{A}=\left\{\alpha \in \mathbb{N}_{0}: \alpha \leq m\right\} .
$$

Corollary 2.2. Let $X$ and $Y$ be complex Banach spaces. Let $\Omega \subset X$ be a complete circular (balanced) domain and $D \subset Y$ be a convex domain. Suppose that $f \in \operatorname{Hol}(\Omega, D)$ is a holomorphic mapping and

$$
f(z)=\sum_{k=0}^{\infty} P_{k}(z)
$$

is its series of homogeneous polynomials. Then for each $m \in \mathbb{N}$ the partial sum $S_{m}$ defined by

$$
S_{m}(z):=\sum_{k=0}^{m} P_{k}(z)
$$


maps $r_{m} \Omega$ into $D$, where $r_{1}=1 / 2, r_{2}=\sqrt{3 / 8}$ and $r_{m}, m \geq 3$, is the unique positive solution of (2.9).

Thus, Theorem 2.1 follows. If, in particular, we let in Corollary 2.2 the Banach spaces be one-dimensional, $X=Y=\mathbb{C}^{1}, \Omega=\Delta$ and $D=\Pi:=$ $\{\lambda \in \mathbb{C}: \operatorname{Re} \lambda>0\}$ be the right-half plane in the complex plane $\mathbb{C}$, then we obtain the following one-dimensional result, which will be used in what follows.

Corollary 2.3. Let $f \in \operatorname{Hol}(\Delta, \Pi)$. Denote by $f(\lambda)=\sum_{k=0}^{\infty} a_{k} \lambda^{k}$ its Taylor series. Then for each partial sum $S_{m}(\lambda)=\sum_{k=0}^{m} a_{k} \lambda^{k}$ we have $S_{m}\left(r_{m} \lambda\right) \in \Pi$ for all $\lambda$ with $|\lambda|<1$, where $r_{m}$ is the unique positive solution of $(2.9)$.

3. Applications. Let $X$ be a complex Banach space, and $\Omega$ be a domain in $X$. For a given holomorphic mapping $f \in \operatorname{Hol}(\Omega, X)$ consider the following Cauchy problem:

$$
\left\{\begin{array}{l}
\frac{\partial F(t, z)}{\partial t}+f(F(t, z))=0 \\
F(0, z)=z, \quad z \in \Omega .
\end{array}\right.
$$

It is clear that for any initial point $z \in \Omega$ the problem (3.13) has a local solution.

Definition 3.1. The mapping $f \in \operatorname{Hol}(\Omega, X)$ is said to be a generator in $\Omega$ if for any point $z_{0} \in \Omega$, the Cauchy problem (3.13) has a unique solution $\{F(t, z)\} \subset \Omega$ defined on $\mathbb{R}^{+} \times \Omega$.

In this situation the solution $F(t, \cdot)$ is a semigroup of holomorphic selfmappings of the domain $\Omega \subset X$. The family of all holomorphic generators on $\Omega$ will be denoted by $\mathcal{G}(\Omega)$. This set is a real cone in $\operatorname{Hol}(\Omega, X)$ (see $[16])$.

In what follows $\Omega$ will be the open unit ball in $X$. One of the standard tools of investigation of differential equations and approximation of its solutions is to replace the mapping $f$ in (3.13) by its Taylor polynomials. Let, as above, $S_{n}$ be the partial sum of the series of homogeneous polynomials for $f$ (see (2.4)). In addition to (3.13), consider the approximate Cauchy problem

$$
\left\{\begin{array}{l}
\frac{\partial F_{n}(t, z)}{\partial t}+S_{n}\left(F_{n}(t, z)\right)=0, \\
F_{n}(0, z)=z, \quad z \in \Omega .
\end{array}\right.
$$

The following questions arise naturally: Does there exist a number $r>0$ such that for each $n \geq 1$, the mapping $S_{n}$ is a generator on $r \Omega$ ? As we shall see below, if $f(0)=0$ then the answer is affirmative. Moreover, the maximal number $r$ such that each $S_{n}, n=1,2, \ldots$, is a generator on $r \Omega$ is 
$r=1 / 2$. Note that, in general, the mapping $f$ itself may not be a generator on balls $r \Omega$.

Theorem 3.1. Let $X$ be a complex Banach space, and let $\Omega \subset X$ be the open unit ball in $X$. Suppose that $f \in \mathcal{G}(\Omega)$ is a holomorphic generator on $\Omega$ with $f(0)=0$. Then for each $n \in \mathbb{N}$ we have $S_{n+1} \in \mathcal{G}\left(r_{n} \Omega\right)$, where $r_{n}$ is the unique solution of $(2.9)$ in $(0,1)$.

Proof. For $z \in X \backslash\{0\}$ denote by $J(z)$ the so-called duality set:

$$
J(z):=\left\{z^{*} \in X^{\prime}: \operatorname{Re}\left\langle z, z^{*}\right\rangle=\|z\|^{2}=\left\|z^{*}\right\|^{2}\right\} .
$$

The following criterion for $f$ to belong to $\mathcal{G}(\Omega)$ has been proved in [1]:

$$
\operatorname{Re}\left\langle f(z), z^{*}\right\rangle \geq \operatorname{Re}\left\langle f(0), z^{*}\right\rangle\left(1-\|z\|^{2}\right) \quad \text { for all } z \in \Omega, z^{*} \in J(z),
$$

and is called the flow invariance condition. In the case $f(0)=0$ this inequality reads

$$
\operatorname{Re}\left\langle f(z), z^{*}\right\rangle \geq 0 \quad \text { for all } z \in \Omega, z^{*} \in J(z) .
$$

For a fixed $z \in \Omega$ define a holomorphic function $p \in \operatorname{Hol}(\bar{\Delta}, \mathbb{C})$ by

$$
p(\lambda):=\frac{1}{\lambda}\left\langle f(\lambda z), z^{*}\right\rangle=\sum_{k=0}^{\infty}\left\langle P_{k+1}(z), z^{*}\right\rangle \lambda^{k} .
$$

(This function is well defined on $\Omega$ because $f(0)=0$.) For all $\lambda$ with $|\lambda|=1$, by (3.15) we have

$$
\operatorname{Re} p(\lambda)=\operatorname{Re} \bar{\lambda}\left\langle f(\lambda z), z^{*}\right\rangle=\operatorname{Re}\left\langle f(\lambda z),(\lambda z)^{*}\right\rangle \geq 0 .
$$

Thus, $\operatorname{Re} p(\lambda) \geq 0$ for all $\lambda \in \bar{\Delta}$. Denote by $p_{n}$ the Taylor polynomial of $p$ :

$$
p_{n}(\lambda)=\sum_{k=0}^{n}\left\langle P_{k+1}(z), z^{*}\right\rangle \lambda^{k} .
$$

By Corollary 2.1 and the continuity of $p$ we get

$$
\operatorname{Re} p_{n}\left(r_{n}\right) \geq 0 \text {. }
$$

Now we note that

$$
\begin{aligned}
\left\langle S_{n+1}\left(r_{n} z\right),\left(r_{n} z\right)^{*}\right\rangle & =\sum_{k=0}^{n}\left\langle P_{k+1}\left(r_{n} z\right),\left(r_{n} z\right)^{*}\right\rangle \\
& =\sum_{k=0}^{n}\left\langle P_{k+1}(z), z^{*}\right\rangle r_{n}^{k+2}=p_{n}\left(r_{n}\right) r_{n}^{2} .
\end{aligned}
$$

Since $z \in \Omega$ is arbitrary, the last two inequalities imply that

$$
\operatorname{Re}\left\langle S_{n+1}(z),(z)^{*}\right\rangle \geq 0 \quad \text { for all } z \in \overline{r_{n} \Omega} .
$$

Then, by a result of Martin [15], we conclude that $S_{n+1} \in \mathcal{G}\left(r_{n} \Omega\right)$. 


\section{References}

[1] D. Aharonov, S. Reich and D. Shoikhet, Flow invariance conditions for holomorphic mappings in Banach spaces, Math. Proc. Roy. Irish Acad. 99A (1999), 93-104.

[2] L. Aizenberg, Multidimensional analogues of Bohr's theorem on power series, Proc. Amer. Math. Soc. 128 (2000), 1147-1155.

[3] - Bohr theorem, in: Encyclopaedia of Mathematics. Suppl. II, Kluwer, Dordrecht, 2000, 2975-2979.

[4] -, A generalization of Carathéodory's inequality and the Bohr radius for multidimensional power series, Complex Variables, to appear.

[5] L. Aizenberg, A. Aytuna and P. Djakov, An abstract approach to Bohr's phenomenon, Proc. Amer. Math. Soc. 128 (2000), 2611-2619.

[6] - - - - Generalization of a theorem of Bohr for bases in spaces of holomorphic functions of several complex variables, J. Math. Anal. Appl. 258 (2001), 429-447.

[7] L. A. Aizenberg, I. B. Grossman and Yu. F. Korobeinik, Some remarks on the Bohr radius for power series, Izv. Vyssh. Uchebn. Zaved. Mat. 2002, no. 10, 3-10 (Russian).

[8] L. Aizenberg, E. Liflyand and A. Vidras, Multidimensional analogue of the van der Corput-Visser inequality and its application to the estimation of the Bohr radius, Ann. Polon. Math. 80 (2003), 47-54.

[9] L. Aizenberg and N. Tarkhanov, A Bohr phenomenon for elliptic equations, Proc. London Math. Soc. 82 (2001), 385-401.

[10] H. P. Boas, Majorant series, J. Korean Math. Soc. 37 (2000), 321-337.

[11] H. P. Boas and D. Khavinson, Bohr's power series theorem in several variables, Proc. Amer. Math. Soc. 125 (1997), 2975-2979.

[12] H. Bohr, A theorem concerning power series, Proc. London Math. Soc. 13 (1914), $1-5$.

[13] P. B. Djakov and M. S. Ramanujan, A remark on Bohr's theorem and its generalizations, J. Analysis 8 (2000), 65-77.

[14] E. Landau und D. Gaier, Darstellung und Begründung einiger neuerer Ergebniße der Funktionentheorie, Springer, 1986.

[15] R. H. Martin, Jr., Differential equations on closed subsets of a Banach space, Trans. Amer. Math. Soc. 179 (1973), 399-414.

[16] S. Reich and D. Shoikhet, Generation theory for semigroups of holomorphic mappings in Banach spaces, Abstr. Appl. Anal. 1 (1996), 1-44.

[17] W. Rogosinski, Über Bildschranken bei Potenzreihen und ihren Abschnitten, Math. Z. 17 (1923), 260-276.

[18] I. Schur und G. Szegő, Über die Abschnitte einer im Einheitskreise beschränkten Potenzreihe, Sitz.-Ber. Preuss. Akad. Wiss. Berlin Phys.-Math. Kl. 1925, 545-560.

Department of Mathematics

Bar-Ilan University

52900 Ramat-Gan, Israel

E-mail: aizenbrg@macs.biu.ac.il
Department of Mathematics ORT Braude College 21982 Karmiel, Israel

E-mail: elin_mark@hotmail.com davs27@netvision.net.il

Received May 4, 2004

Revised version July 9, 2004 